\section{Red blood cell distribution width and erythrocyte parameters in patients with brain injury after mild head trauma}

\author{
Giuseppe Lippi, ${ }^{1}$ Andrea Carbucicchio, ${ }^{2}$ \\ Paola Avanzini, ${ }^{1}$ Mariella Dipalo, ${ }^{1}$ \\ Mario Benatti, ${ }^{2}$ Gianfranco Cervellin ${ }^{2}$ \\ 'Laboratory of Clinical Chemistry and \\ Hematology, Pathology and Laboratory \\ Medicine Department, Parma University \\ Hospital; 'Emergency Department, \\ Parma University Hospital, Italy
}

\section{Abstract}

This prospective study was planned to assess whether red blood cell (RBC) parameters may be useful in diagnostics of patients with brain injury after mild head trauma. The $\mathrm{RBC}$ count, hemoglobin, hematocrit, RBC distribution width (RDW) and mean corpuscular volume were assessed in all consecutive patients admitted to the emergency department over 3 months with isolate, mild head trauma and Glasgow coma scale between 1415 , and seen within $3 \mathrm{~h}$ from trauma. The final study population consisted of 54 patients (21 women and 33 men; median age $=48$ years), of whom, 13 (24\%) with positive computed tomography (CT). No significant difference was found for age $(\mathrm{P}=0.45)$ and gender $(\mathrm{P}=0.21)$ distribution between $\mathrm{CT}$ positive and negative patients. No significant difference was observed for the median concentration of all the RBC parameters tested, and the prevalence of anemia $(\mathrm{P}=0.37)$ and anysocytosis $(\mathrm{P}=0.40)$ did not differ significantly between patients with positive and negative CT. Red blood cell distribution width assessment upon patient admission did not provide a significant contribution to final diagnosis of mild head injury in receiver operating characteristic curve analysis [area under the curve (AUC) $0.51 ; \mathrm{P}=0.44]$. We conclude that assessment of RDW does not provide useful clinical information for diagnosing brain injury after mild head trauma.

\section{Introduction}

Head trauma is an important cause of death and disability worldwide, with an annual incidence of approximately 600 patients per 100,000 , and a mortality rate of 17 cases per $100,000 .^{1}$ Among all emergency department
(ED) admissions for head trauma, mild head injury (MHI) is approximately 15 - and 20-time more frequent than moderate and severe head injury, respectively. ${ }^{1}$

The diagnostics of brain injury after mild head trauma is challenging. Most national and international guidelines still recommend the use of imaging techniques, namely computed tomography (CT) scanning of the head. ${ }^{2}$ These techniques, however, carry several drawbacks, such as high costs as well as the health risk related to harmful ionizing radiation. ${ }^{3}$ It is also noteworthy that the overall diagnostic efficiency of CT is low, since a minority of patients (i.e. less than 1/4) admitted to the ED with MHI really have intracranial injuries, and even fewer of them finally require neurosurgery (i.e. less than $2 \%){ }^{4}$ The use of additional diagnostic tools is thereby advisable for triaging patients with MHI, especially in the ED where early and accurate diagnosis is necessary to reduce staying and contextually improve quality of care. ${ }^{5}$ In this perspective, the introduction of biomarkers of brain injury represents an appealing perspective due to the relatively low harm caused by venipuncture, the healthcare savings as compared with more expensive imagining techniques, and the rapid turnaround time enabled by the current laboratory methods. Some biological markers of brain injury have recently been proposed, including protein S100B, neuron-specific enolase (NSE), glial fibrillary acidic protein (GFAP), myelin basic protein (MBP), cleaved Tau protein (CTP), brain type fatty acid-binding protein (B-FABP), kallikrein 6 (KLK6), ubiquitin C-terminal hydrolase (UCH-L1) and II-spectrin breakdown product $145 \mathrm{kDa}$ (SBDP145), among others. ${ }^{6-10}$ Although encouraging results have been published about the potential usefulness of some of these biomarkers in research studies, especially protein $\mathrm{S} 100 \mathrm{~B},{ }^{11,12}$ there is still limited clinical evidence to allow recommendation of routine implementation for diagnosis and management of patients with MHI. ${ }^{13,14}$

Red blood cell (RBC) distribution width (RDW) is a measure of anysocytosis, which is commonly used in combination with mean corpuscular volume (MCV) for investigating the underlying cause(s) of anemia. It is typically calculated by dividing the standard deviation (SD) of RBC volume by the MCV and multiplying it by 100 , for finally expressing the result as a percentage. ${ }^{15}$ Several lines of evidence now attest that abnormal values of this simple and inexpensive parameter, which is automatically generated by all modern hemocytometers along with the complete blood count, reflect a kaleidoscope of underlying pathological conditions such as inflammation, ${ }^{16}$ metabolic imbalances $^{17,18}$ or tissue injury, ${ }^{19}$ so that its assessment may be helpful in the diagnostic approach of several human disorders such as
Correspondence: Giuseppe Lippi, Laboratory of Clinical Chemistry and Hematology, Pathology and Laboratory Medicine Department, Parma University Hospital, via Gramsci 14, 43126 Parma, Italy.

Tel. +39.0521 .703050 - Fax: +39.0521 .703791$

E-mail: glippi@ao.pr.it, ulippi@tin.it

Key words: erythrocyte, red blood cell, red blood cell distribution width, head trauma, brain injury.

Contributions: the authors contributed equally.

Conflict of interests: the authors declare no potential conflict of interests.

Received for publication: 19 April 2013

Revision received: 5 June 2013.

Accepted for publication: 5 June 2013.

This work is licensed under a Creative Commons Attribution 3.0 License (by-nc 3.0)

(C) Copyright G. Lippi et al., 2013

Licensee PAGEPress, Italy

Emergency Care Journal 2013; 9:e13

doi:10.4081/ecj.2013.e13

acute myocardial infarction, ${ }^{20}$ pulmonary embolism, ${ }^{21}$ acute infections, ${ }^{22}$ and even cancer. $^{23}$ Recent studies have also emphasized that the value of RDW may be significantly increased in patients with some neurological disorders, such as Alzheimer's disease ${ }^{24}$ reactive amyloidosis, ${ }^{25}$ as well as acute cerebral infarction. ${ }^{26,27}$ In these cases, thus, it may be used as a diagnostic and prognostic index. However, since this parameter has not been investigated in patients with head trauma to the best of our knowledge, we planned a prospective study with the aim to assess whether its measurement may be useful in the diagnostics of brain injury after mild head trauma.

\section{Materials and Methods}

We planned a prospective study, including all consecutive patients aged 14 to 80 years, admitted to the ED of Parma University Hospital (Italy) between September and November 2012. Patients had isolate, mild head trauma, Glasgow coma scale (GCS) between 14 and 15 , and were visited within $3 \mathrm{~h}$ from trauma (total number of consecutive eligible patients $=54$ ). The Parma University Hospital is identified as a level 2 trauma center, and the volume of ED visits is about 90,000 per year. All patients met the local criteria for mild head trauma requiring CT scanning: GCS 14-15; history of loss of consciousness associated with at least one of i) peritraumatic 
amnesia, ii) previous neurosurgical procedure, iii) inherited coagulopathy or anticoagulant therapy, iv) vomit (more than 1 episode), v) epilepsy or post-traumatic seizures, and vi) worsening headache; and clinical findings of depressed skull fracture, basilar skull fracture, focal neurological abnormalities and drug or alcohol intoxication. Unenhanced CT scanning was performed in all patients, within $3 \mathrm{~h}$ after occurrence of trauma, with 16-slice Siemens Somatom Emotion (Siemens AG, Munich, Germany) scanner with sequential slicing 4.8 $\mathrm{mm}$ for brain parenchyma and 1.2 or $2.4 \mathrm{~mm}$ for cranial vault and base. The presence of any intracranial pathology associated with brain injury (i.e. cerebral contusion, swelling, traumatic subarachnoid haemorrhage, acute subdural, epidural or parenchymal hematoma) was considered as a positive CT result. Blood samples were collected immediately after patient arrival at the ED. The main erythrocyte parameters, including RBC count, hemoglobin, hematocrit, MCV and RDW, were assessed on Sysmex XE-2100 (Sysmex Inc., Kobe, Japan; commercialized in Italy by Dasit SpA, Cornaredo). Anemia was defined according to the World Health Organization (WHO) criteria as hemoglobin value $<120 \mathrm{~g} / \mathrm{L}$ in women and $<130 \mathrm{~g} / \mathrm{L}$ in men, whereas anysocytosis was defined as RDW value $>14.0 \%$. Data were shown together with the median and interquartile range (IQR). The significance of differences between the groups was assessed by Mann-Whitney U test (for continuous variables) and the chi-squared test (for categorical variables). Diagnostic performance of $\mathrm{RBC}$ parameters was also tested by means of receiver operating characteristic (ROC) curve analysis. Statistical analysis was performed with Analyse-it for Microsoft Excel (Analyse-it Software Ltd., Leeds, UK). The study was carried out in accordance with the Declaration of Helsinki, under the terms of all relevant local legislation.

\section{Results}

The final, eligible study population consisted of 54 patients (median age 48 years, IQR $32-69$ years; 21 women and 33 men), of whom 13 (24\%) with positive CT. No significant difference was found for age $(\mathrm{P}=0.45)$ and gen$\operatorname{der}(\mathrm{P}=0.21)$ distribution between $\mathrm{CT}$ positive and negative patients. As shown in Table 1, no significant difference was either found for the median concentration of all the RBC parameters tested, including RDW (Figure 1). The prevalence of anemia (i.e. 23 vs $27 \% ; \mathrm{P}=0.37$ ) and anysocytosis (i.e. 23 vs $20 \%$; $\mathrm{P}=0.40$ ) did not differ significantly in patients with positive CT than in those with negative CT. It is also noteworthy that the assessment of RDW upon patient admission did not provide a significant contribution to the final diagnosis of MHI, as attested by the poor performance of the ROC curve [i.e. area under the curve (AUC) $0.51 ; 95 \%$ confidence interval (CI), 0.33 to $0.69 ; \mathrm{P}=0.44$ ].

\section{Discussion}

Red blood cell distribution width is an inexpensive, routinely reported test, that is strongly emerging as a powerful predictor of death and disability in general population, as well as in patients with various disorders. ${ }^{19}$ Some previous clinical investigations have shown that RDW may be a useful diagnostic and/or prognostic index in patients with neurological diseases. It has been reported that the mean RDW value was significantly higher in patients with Alzheimer's disease than in controls, also showing a negative correlation with minimental state examination (MMSE) ${ }^{24}$ and in patients with reactive amyloidosis. ${ }^{25}$ Even more interestingly, Ani and Ovbiagele analyzed data from the national health and nutrition examination survey (NHANES), to establish whether any relationship between RDW and mortality in patients with stroke exists. ${ }^{26}$ The mean RDW was found to be significantly increased in patients with stroke that in those without (i.e. 13.7 vs $13.2 \% ; \mathrm{P}<0.01$ ), and the RDW value was higher in patients with stroke who later died as compared with those who continued to live (13.9 vs $13.4 \% ; \mathrm{P}<0.01)$. Subjects with elevated RDW (fourth $v s$ first quartile) were also more likely to have experienced a stroke [odds ratio (OR) 1.71], whereas RDW in the top $v s$ the bottom quartile independently predicted subsequent cardiovascular and all-cause deaths, with hazard ratios of 2.38 and 2.0, respectively. More recently, Kim et al. assessed the potential association of RDW with poor functional outcome and all-cause mortality at three months, as well as survival time one year after stroke, in 847 consecutive patients admitted to the ED with a first-ever acute cerebral infarction. ${ }^{27}$ The results of multivariate logistic regression showed that increased values of RDW were independently associated with poor functional outcome (OR 1.22 per $1 \%$ increment in RDW) and all-cause

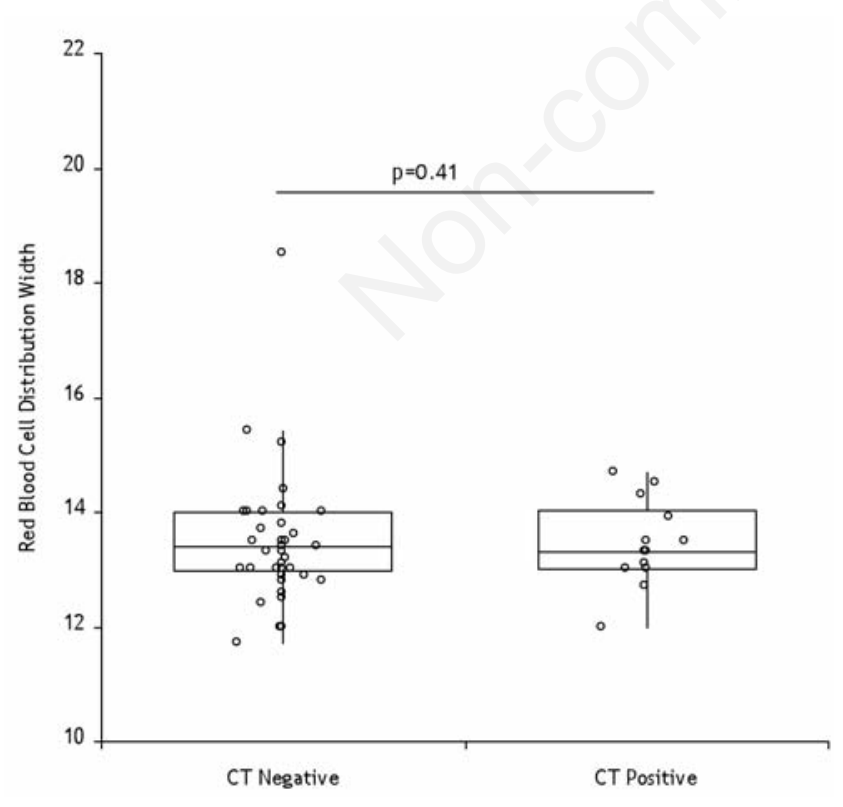

Figure 1. Distribution of median and interquartile range values of red blood cell distribution width in patients with mild head injury. Positive or negative designate, respectively, the presence or lack of brain injury after mild head trauma with computer tomography evaluation. The boxes are drawn at the median value (horizontal line) and interquartile range.
Table 1. Distribution of median and interquartile range values of red blood cell count, hematocrit, hemoglobin, mean corpuscular volume and red blood cell distribution width in patients with mild head injury.

\begin{tabular}{lccc} 
& & & \\
& Negative $^{\circ}$ & Positive $^{\circ}$ & P \\
No. of patients & - & 13 & - \\
RBC (109/L) & $4.71(4.25-5.05)$ & $4.74(4.27-5.11)$ & 0.42 \\
\hline Hematocrit (\%) & $42.0(38.4-44.2)$ & $41.8(38.4-45.3)$ & 0.29 \\
Hemoglobin (g/L) & $136(127-147)$ & $135(129-148)$ & 0.45 \\
\hline MCV (fL) & $89.8(86.0-93.6)$ & $89.9(85.2-92.2)$ & 0.45 \\
RDW (\%) & $13.4(13.0-14.0)$ & $13.3(13.0-14.0)$ & 0.41 \\
\hline
\end{tabular}

CT, computer tomography; RBC, red blood cell; MCV, mean corpuscular volume; RDW, RBD distribution width. ${ }^{\circ}$ Positive or negative designate, respectively, the presence or lack of brain injury after mild head trauma with CT evaluation. 
death (OR 1.39 per $1 \%$ increment in RDW) at 3 months. The value of RDW was also found to be independent predictor of survival, with a hazard ratio of 1.33 per $1 \%$ increment in RDW.

\section{Conclusions}

Taken together, earlier studies support the hypothesis that RDW assessment may be of clinical value in patients with neurological disorders, and have prompted us to investigate whether it may also have a role for diagnostic evaluation of patients admitted to ED with mild head trauma. As clearly shown in Table 1 and Figure 1, however, we failed to find any statistically significant difference in RDW and other RBC parameters for identifying the presence of MHI in our prospective investigation. The likelihood of anemia and anysocytosis was also comparable between patients with and without intracranial pathologies associated with brain injury. Hence, despite the limited number of patients studied, we conclude that assessment of RDW does not provide useful clinical information in this setting.

\section{References}

1. Fabbri A, Servadei F, Marchesini G, et al. The changing face of mild head injury: temporal trends and patterns in adolescents and adults from 1997 to 2008. Injury 2010;41:913-7.

2. Tavender EJ, Bosch M, Green S, et al. Quality and consistency of guidelines for the management of mild traumatic brain injury in the emergency department. Acad Emerg Med 2011;18:880-9.

3. Hall EJ, Brenner DJ. Cancer risks from diagnostic radiology. Brit J Radiol 2008;81: $362-78$.

4. af Geijerstam JL, Oredsson S, Britton M. Medical outcome after immediate computed tomography or admission for observation in patients with mild head injury: randomised controlled trial. Brit Med J 2006; 333:465-9.

5. McCarthy ML. Overcrowding in emergency departments and adverse outcomes. Brit
Med J 2011;342:d2830.

6. Korfias S, Papadimitriou A, Stranjalis G, et al. Serum biochemical markers of brain injury. Mini-Rev Med Chem 2009;9:227-34.

7. Bayani J, Diamandis EP. The physiology and pathobiology of human kallikrein-related peptidase 6 (KLK6). Clin Chem Lab Med 2011;50:211-33.

8. Berger RP, Hayes RL, Richichi R, et al. Serum concentrations of ubiquitin C-terminal hydrolase-L1 and $\alpha$ II-spectrin breakdown product $145 \mathrm{kDa}$ correlate with outcome after pediatric TBI. J Neurotraum 2012;29:162-7.

9. Okonwo D0, Yue JK, Puccio AM et al. GFAP-BDP as an acute diagnostic marker in traumatic brain injury: results from the prospective TRACK-TBI study. J Neurotraum 2013 Mar 14 (Epub ahead of print).

10. Lippi G, Cervellin G. Diagnostic approach to the mild head trauma of the adult in emergency medicine: between biomarkers and imaging. Recenti Prog Med 2013;104: 120-32.

11. Cervellin G, Benatti M, Carbucicchio A, et al. Serum levels of protein S100B predict intracranial lesions in mild head injury. Clin Biochem 2012;45:408-11.

12. Zongo D, Ribéreau-Gayon R, Masson F, et al. S100-B protein as a screening tool for the early assessment of minor head injury. Ann Emerg Med 2012;59:209-18.

13. Schiavi P, Laccarino C, Servadei F. The value of the calcium binding protein $\mathrm{S} 100$ in the management of patients with traumatic brain injury. Acta Biomed 2012;83:520.

14. Tavarez MM, Atabaki SM, Teach SJ. Acute evaluation of pediatric patients with minor traumatic brain injury. Curr Opin Pediatr 2012;24:307-13.

15. Montagnana M, Cervellin G, Meschi T, et al. The role of red blood cell distribution width in cardiovascular and thrombotic disorders. Clin Chem Lab Med 2011;50: 635-41.

16. Lippi G, Targher G, Montagnana M, et al. Relation between red blood cell distribution width and inflammatory biomarkers in a large cohort of unselected outpatients. Arch Pathol Lab Med 2009;133:628-32.

17. Lippi G, Targher G, Montagnana M, et al.
Relationship between red blood cell distribution width and kidney function tests in a large cohort of unselected outpatients. Scand J Clin Lab Inv 2008;68:745-8.

18. Milić S, Mikolasević I, Radić M, et al. Clinical utility of red cell distribution width in alcoholic and non-alcoholic liver cirrhosis. Collegium Antropol 2011;35 (Suppl.2):335-8.

19. Patel KV, Semba RD, Ferrucci L, et al. Red cell distribution width and mortality in older adults: a meta-analysis. J Gerontol ABiol 2010;65:258-65.

20. Lippi G, Filippozzi L, Montagnana M, et al. Clinical usefulness of measuring red blood cell distribution width on admission in patients with acute coronary syndromes. Clin Chem Lab Med 2009;47:353-7.

21. Ozsu S, Abul Y, Gunaydin S, et al. Prognostic value of red cell distribution width in patients with pulmonary embolism. Clin Appl Thromb-Hem 2012 Nov 8 (Epub ahead of print).

22. Lippi G, Dipalo M, Teti L, et al. Relationship between red blood cell distribution width and prognostic biomarkers in patients admitted to the emergency department with acute infections. Eur J Intern Med 2013;24:e15-6.

23. Baicus C, Caraiola S, Rimbas M, et al. Utility of routine hematological and inflammation parameters for the diagnosis of cancer in involuntary weight loss. $\mathrm{J}$ Invest Med 2011;59:951-5.

24. Öztürk ZA, Ünal A, Yiğiter R, et al. Is increased red cell distribution width (RDW) indicating the inflammation in Alzheimer's disease (AD)? Arch Gerontol Geriat 2013;56:50-4.

25. Erdem E, Erdem D, Dilek M, et al. Red cell distribution width and mean platelet volume in amyloidosis. Clin Appl Thromb-Hem 2012 0ct 17 (Epub ahead of print).

26. Ani C, Ovbiagele B. Elevated red blood cell distribution width predicts mortality in persons with known stroke. J Neurol Sci 2009;277:103-8.

27. Kim J, Kim YD, Song TJ, et al. Red blood cell distribution width is associated with poor clinical outcome in acute cerebral infarction. Thromb Haemostasis 2012;108: 349-56. 